\title{
Fault Prediction Algorithm for Multiple Mode Process Based on Reconstruction Technique
}

\author{
Jie Ma and Jianan Xu \\ School of Automation, Beijing Information Science and Technology University, Beijing 100192, China \\ Correspondence should be addressed to Jie Ma; mjbeijing@163.com
}

Received 29 October 2014; Accepted 21 December 2014

Academic Editor: Gang Li

Copyright (C) $2015 \mathrm{~J}$. Ma and J. Xu. This is an open access article distributed under the Creative Commons Attribution License, which permits unrestricted use, distribution, and reproduction in any medium, provided the original work is properly cited.

\begin{abstract}
In the framework of fault reconstruction technique, this paper studies the problems of multiple mode process fault detection, fault estimation, and fault prediction systematically based on multi-PCA model. First, a multi-PCA model is used for fault detection in steady state process under different conditions, while a weighted algorithm is applied to transition process. Then, describe the faults quantitatively and use the optimization method to derive the fault amplitude under the sense of fault reconstruction. Fault amplitude drifts under different conditions even if the same fault occurs. To solve the above problem, consistent estimation algorithm of fault amplitude under different conditions has been studied. Last, employ the support vector machine (SVM) to predict the trend of the fault amplitude. Effectiveness of the algorithms proposed in this paper has been verified using Tennessee Eastman process as the study object.
\end{abstract}

\section{Introduction}

Modern engineering systems become more and more complex while the scale becomes larger and larger simultaneously. However, the operation safety of complex systems is inversely proportional to their scales. Fault diagnosis and prediction techniques offer an important way to improve the operation safety of complex engineering systems, which are often operating under multiple mode process due to the following reasons: changes of the nature of raw materials, external environment disturbances, drifting of the load in a process and even equipment aging, and so forth. All of the factors mentioned above may lead to the difference between the practical operating processes and the rated operating processes; otherwise equipment itself may have a plurality of operating periods due to the adjustment of production programs. For example, a ship sailing task needs to go through several stages from set sail, offshore sailing, far-shore sailing, and returning back to the harbor. Operating condition of the marine system changes frequently during the sailing. And a marine system may have more than one working condition even in the same stage. Therefore, the monitoring technology of multicondition process has gradually achieved widespread attention both in industry and in academia [1].
With the wide application of distributed control system (DCS) in industrial processes, massive process data associated with the operating status of the device can easily be saved. Since the 1990s, the data-driven multivariate statistical monitoring technology has been successfully applied in industrial processes [2]. Traditional multivariate statistical monitoring techniques include methods based on PCA and PLS. The traditional methods are based on the assumption that the process data obey Gaussian distribution, the data must be linear, and the process must be stable with only one operating condition, and so forth. However, the practical industrial process data often do not strictly obey the Gaussian distribution and also are usually nonlinear, time-varying, multiconditioned, and dynamic. So if the traditional methods are being used to monitor those practical processes, it will inevitably lead to inaccuracy analysis of process performance as well as false alarm and missing alarm of process failures [3]. For multiple mode process, improved methods have been proposed based on the traditional PCA/PLS, which are mainly divided into recursive iterative method and multimodel method. The basic idea of recursive iteration method is to add new process data into the modeling data matrix continuously. By updating the model parameters, the 
model can adapt to new conditions [4]. The recursive iteration method is used relatively less in practical applications, because the method cannot distinguish changes during normal operating conditions and fault conditions correctly and is more dependent on the process mechanism and knowledge. The basic idea of multimodel method is to divide different operating conditions first by clustering algorithm and then use process data for each condition to establish submodels. Finally, construct a global detection indicator to monitor the process data. Multiple PCA model is studied in [5-7], super PCA model is studied in [8], probabilistic principal component analysis (PPCA) is studied in [9], adjacent PCA model is studied in [10], PCA model based on Bayesian classifier is studied in [11-13], mixed PCA model is studied in [14], Gaussian mixture model (GMM) is studied in [15], and so on.

Multiple mode process switches constantly between "steady state mode 1 transition process-steady state mode 2 " and the fault detection of steady state modes should be considered and also the fault detection of the transition process should be studied. For example, in the literature [16], Lu et al. used "hard partition" to obtain the transition region between steady state modes. In the literature [17], Zhao et al. and another literature [18], Yao and Gao further proposed the concept of "soft partition" and separate the data of the transition region and the data of the stable region preferably.

Currently, fault detection and fault diagnosis of multiple mode process have achieved remarkable achievements while the research of fault prediction is still rare $[19,20]$. This paper proposed a fault prediction method of multiple mode process based on fault reconstruction technology. First, multiple PCA models are applied to fault detection of multiple mode process. Then, fault reconstruction technology is used to estimate the fault amplitude. Last, employ the support vector machine (SVM) to predict the trend of the fault amplitude. The data of Tennessee Eastman process is applied to verify the validity of the algorithm.

\section{Fault Detection Algorithms under Multiple Mode Process}

Multiple mode process includes steady state process and transition process. Build multi-PCA models to adapt to steady state processes of different conditions and calculate statistics of the corresponding detection indicator Hotelling's $T^{2}$ and SPE (squared prediction error) of each PCA model for fault detection. For transition processes, a weighted method is applied to calculate statistics and control limits for fault detection.

Define $\mathbf{x} \in R^{m}$ that represents a sample vector with $m$ measured variables, and there are $n$ samples during operation. The data matrix $\mathbf{X} \in R^{n \times m}$ is composed by $n$ samples, in which each row represents a sample and each column represents that a measured variable includes $n$ samplings. First, transfer each column of the data matrix $\mathbf{X}$ to zero means and unit variance variable through standardized processing; then, after standardization, the covariance matrix of sample $\mathbf{x}$ can be obtained:

$$
\mathbf{S}=\operatorname{cov}(\mathbf{x}) \approx \frac{1}{n-1} \mathbf{X}^{T} \mathbf{X}
$$

Then analyse the eigenvalues of the covariance matrix and arrange the eigenvalues in descending order. Each column of the data matrix $\mathbf{X}$ transfers to zero mean and unit variance variable can be obtained as follows: subtract the corresponding variable from each column of $\mathbf{X}$ and then divide by the corresponding variable standard deviation.

The PCA model divides the measured variable space into principal subspace and residual subspace; they are orthogonal and complementary. Any sample vector can be decomposed into projections on principal subspace and residual subspace; that is to say, the PCA model decomposes the sample matrix $\mathbf{X} \in R^{n \times m}$ into two parts: $\widehat{\mathbf{X}}$ and E. Consider

$$
\mathbf{X}=\widehat{\mathbf{X}}+\mathbf{E}=\mathbf{T} \mathbf{P}^{T}+\mathbf{E}
$$

where $\widehat{\mathbf{X}}$ is the modeled part; $\mathbf{E}$ is the residual part; $\mathbf{P} \in R^{m \times A}$ is load matrix, which is made up of the former $A$ eigenvectors of $S$; $A$ is the number of principal elements; $\mathbf{T} \in R^{n \times A}$ is scoring matrix, $\mathbf{T}=\mathbf{X P}$.

The multi-PCA method is to establish a corresponding principal element model according to the historical data of existing measured variables in each steady state operating condition, thus establishing the multiprincipal element model group which contains all operating conditions; namely,

$$
\mathbf{X}^{(q)}=\widehat{\mathbf{X}}^{(q)}+\mathbf{E}^{(q)}
$$

where $q$ is the number of stable conditions.

2.1. Fault Detection of Steady State Process. In multi-PCA model, it is needed to calculate the statistics of the corresponding detection indicator $T^{2}$ and SPE for each separate PCA model, that is, $T^{2(q)}$ and $\mathrm{SPE}^{(q)}$. SPE is used to measure the changes of sample vector projections on residual subspace, and $T^{2}$ is used to measure the changes of measured variables on principal subspace. SPE is given as

$$
\mathrm{SPE}=\left\|\left(\mathbf{I}-\mathbf{P P}^{T}\right) \mathbf{x}\right\|^{2} \leq \delta_{\alpha}^{2}
$$

where $\delta_{\alpha}^{2}$ is the control limit of SPE when confidence level is $\alpha$. When SPE is in control limit, the device is running in normal state; however, when SPE is beyond the control limit, a failure occurs. The change of SPE represents the change of correlation between the data. $\delta_{\alpha}^{2}$ is given as

$$
\delta_{\alpha}^{2}=\theta_{1}\left(\frac{c_{\alpha} \sqrt{2 \theta_{2} h_{0}^{2}}}{\theta_{1}}+1+\frac{\theta_{2} h_{0}\left(h_{0}-1\right)}{\theta_{1}^{2}}\right)^{1 / h_{0}}
$$

where $\theta_{i}=\sum_{j=A+1}^{m} \lambda_{j}^{i}(i=1,2,3), h_{0}=1-2 \theta_{1} \theta_{3} / 3 \theta_{1}^{2}$, and $\lambda_{j}$ is the eigenvalue of covariance matrix $\sum$ corresponding to sample matrix X. $c_{\alpha}$ is the threshold value of standard normal 
distribution when confidence level is $\alpha . m$ is the dimension of sample $\mathbf{x}$.

$T^{2}$ is given as

$$
T^{2}=\mathbf{x}^{T} \mathbf{P} \Lambda^{-1} \mathbf{P}^{T} \mathbf{x} \leq T_{\alpha}^{2},
$$

where $\Lambda=\operatorname{diag}\left\{\lambda_{1}, \ldots, \lambda_{A}\right\}$ and $T_{\alpha}^{2}$ is the statistical limit of $T^{2}$ when confidence level is $\alpha$. When $T^{2}$ is in control limit, the device is running in a normal state.

2.2. Fault Detection of Transition Process. In multiple mode process, when a production process switches from one steady state to another, it will go through a slow-changing transition process and cause false alarms of a specific fault if the fault detection method for steady state process is being used in transition process. This paper uses a weighted method that evolves with time to optimize each of $T^{2}$ and SPE of each separate PCA model. Then use the optimized $T^{2}$ and SPE for fault detection. Thus false alarms of a fault during the transition process can be effectively avoided.

When performing an average in statistics, the value which gives some elements more "weight" or influence on the result than other elements in the same set is called weight. Weighted algorithm is the weight multiplied by the value of the corresponding element and then divided by the sum of every weight. In the instant of a steady state condition just to transfer to the next condition, the former condition has a greater impact on the characteristics of the transition process. With time passing, the characteristics of the transition process become more and more close to the next condition. Therefore, during the transition process the value of weights should be time changing. The weight of the former condition transfers from 1 to 0 ; on the contrary the weight of the new condition transfers from 0 to 1 . The weight of the former condition $w_{1}$ and the weight of the new condition $w_{2}$ are given as

$$
\begin{aligned}
& w_{1}=\frac{t-t_{1}}{\Delta t}, \\
& w_{2}=\frac{t_{2}-t}{\Delta t},
\end{aligned}
$$

where $t_{1}$ is the moment when the transition process begins; $t_{2}$ is the moment when the transition process ends, $\Delta t=t_{2}-t_{1}$; $w_{1}+w_{2}=1$.

Suppose that $t=k, \bar{x}_{1}, \bar{x}_{2}$ represent the mean of measured variables of the two adjacent conditions and $\sigma_{1}$ and $\sigma_{2}$ represent the standard deviation of measured variables of the two adjacent conditions; thus the mean $\bar{x}_{t=k}$ and standard deviation $\sigma_{t=k}$ of the transition process are given as

$$
\begin{aligned}
& \bar{x}_{t=k}=w_{1} \times \bar{x}_{1}+w_{2} \times \bar{x}_{2}, \\
& \sigma_{t=k}=w_{1} \times \sigma_{1}+w_{2} \times \sigma_{2} .
\end{aligned}
$$

The sample data can be pretreated by the weighted mean $\bar{x}_{t=k}$ and standard deviation $\sigma_{t=k}$, so covariance matrix of the sample vector $S_{t=k}$ and load matrix of the transition process
$\mathbf{P}_{t=k}$ can be obtained, and then the optimized SPE and $T^{2}$ can be derived:

$$
\begin{gathered}
\mathrm{SPE}_{t=k}=\left\|\left(\mathbf{I}-\mathbf{P}_{t=k} \mathbf{P}_{t=k}^{T}\right) \mathbf{x}_{t=k}\right\|^{2} \leq \delta_{\alpha t=k}^{2}, \\
T_{t=k}^{2}=\mathbf{x}_{t=k}^{T} \mathbf{P}_{t=k} \Lambda_{t=k}^{-1} \mathbf{P}_{t=k}^{T} \mathbf{x}_{t=k} \leq T_{\alpha=k}^{2} .
\end{gathered}
$$

\section{Fault Amplitude Estimation Based on Reconstruction Technique}

When a fault is detected, the fault amplitude which measures the extent of the current fault can be estimated by fault reconstruction technique. Whether the estimation of amplitude of the same fault is consistent under different conditions is an important problem that multicondition brought. If it is not, it should achieve consistency by employing some specific algorithms.

3.1. The Basic Idea of Fault Reconstruction. Fault reconstruction is to reconstruct the process data and remove the effects of faults. The data reconstructed is within the control limits theoretically and is approximately the normal data. Fault estimation is to estimate the fault amplitude after fault reconstruction. When observed data is missing or is obviously failure, the practical data can be replaced by reconstructed data. Fault reconstruction technology has obtained some achievements in the field of fault diagnosis; refer to [21-30].

Suppose $\mathbf{x}$ represents the data of the detected fault, $\mathbf{x}^{*}$ represents the normal data, $\mathbf{x}^{*}, \mathbf{x} \in\left[x_{1}, x_{2}, \ldots, x_{m}\right]$, $m$ represents the number of sensors, which represents the dimension of each measurement sample, and $\mathbf{f}$ represents the fault amplitude. Then

$$
\mathbf{x}=\mathbf{x}^{*}+\boldsymbol{\Xi f}
$$

where $\boldsymbol{\Xi}$ represents fault subspace, also known as fault direction matrix.

Reconstruct the normal data to eliminate the influence of faults and obtain $x_{i}^{*}$, which is the estimated value of normal data

$$
\mathbf{x}_{i}^{*}=\mathbf{x}-\boldsymbol{\Xi}_{i} \mathbf{f}_{i}
$$

where $\mathbf{f}_{i}$ represents the estimated value of $\mathbf{f}$. In geometric meaning, it is pulling sample $\mathbf{x}$ back to the principal subspace along the fault subspace.

Based on $x_{i}^{*}$ which is the estimation of the normal data after reconstruction, we can have $\mathbf{f}_{i}$ which is the estimation of fault amplitude in the sense of least SPE after reconstruction:

$$
\widehat{f}_{i}=\widetilde{\Xi}^{+} x_{i}
$$

where $\widetilde{\boldsymbol{\Xi}}=(\mathbf{I}-\mathbf{C}) \boldsymbol{\Xi}$ denotes the projection from fault subspace $\boldsymbol{\Xi}$ to residual subspace $\mathbf{C}$.

The SPE after reconstruction is

$$
\operatorname{SPE}\left(\mathbf{x}^{*}\right)=\left\|\widetilde{\mathbf{x}}^{*}\right\|^{2}=\left\|\widetilde{\mathbf{x}}-\widetilde{\Xi} \mathbf{f}_{\mathbf{i}}\right\|^{2} .
$$


Reconstruction means searching $\widehat{\mathbf{f}}_{\mathbf{i}}$ to fit the following equation based on (13)

$$
\widehat{\mathbf{f}}_{\mathbf{i}}=\arg \min \left\|\widetilde{\mathbf{x}}-\widetilde{\Xi} \mathbf{f}_{i}\right\|^{2}=\left(\widetilde{\Xi}_{i}^{T} \widetilde{\Xi}_{i}\right)^{-1} \widetilde{\Xi}_{\mathbf{i}}^{T} \widetilde{x} .
$$

The optimal solution of (14) is

$$
\widehat{\mathbf{f}}_{i}=\widetilde{\Xi}^{+} \widetilde{\mathbf{x}}_{i}=\widetilde{\Xi}^{+} \mathbf{x}_{i}
$$

where $\widetilde{\Xi}^{+}$denotes the Moore-Penrose pseudoinverse of $\widetilde{\Xi}$.

3.2. Consistent Estimation of Multiple Mode Process Fault Amplitude. When a fault occurs in condition 1 and lasts to condition 2 , the fault direction matrix $\Xi$ changes, but the fault magnitude remains unchanged. In theory, for the same fault, the fault estimation should be consistent even in different conditions. But in practical industrial process, the result varies due to data noises and machine interference in different conditions. So the consistent estimation of the same fault under different conditions should be studied.

We assume that

$$
\begin{aligned}
& A=\widehat{\mathbf{f}}_{1} \Xi_{i_{1} 1}^{\prime} \\
& B=\widehat{\mathbf{f}}_{2} \Xi_{i-2}^{\prime},
\end{aligned}
$$

where $\widehat{\mathbf{f}}_{1}$ and $\widehat{\mathbf{f}}_{2}$ denote the amplitude estimation of the same fault in the condition and the next condition separately. $\boldsymbol{\Xi}_{i_{-} 1}$ and $\boldsymbol{\Xi}_{i-2}$ denote the projection matrix on residual subspace of the corresponding fault separately; we suppose that the dimensions of $\Xi_{i_{-1}}$ and $\boldsymbol{\Xi}_{i_{-2}}$ are both $\alpha$.

Based on (16), we can derive the amplitude estimations of the same fault under different conditions:

$$
\begin{aligned}
& \mathbf{f}_{1}^{*}=\text { Ad } \\
& \mathbf{f}_{2}^{*}=\text { Be. }
\end{aligned}
$$

We can derive $\mathbf{d}$ and $\mathbf{e}$ in the way of minimizing $\left\|\mathbf{f}_{1}^{*}-\mathbf{f}_{2}^{*}\right\|$. We define $\mathbf{G}=[\mathbf{A}, \mathbf{B}]$ here, $\mathbf{G} \in \mathbf{R}^{n \times 2 k}$. The singular value decomposition of $\mathbf{G}^{T}$ can be described as

$$
\mathbf{U}^{H} \mathbf{G}^{T} \mathbf{V}=\mathbf{\Sigma},
$$

where $\mathbf{U}$ and $\mathbf{V}$ are both unitary matrix with the corresponding dimensions of $2 k$ and $n, \Sigma$ is diagonal matrix, and the diagonal elements are singular values. Sort the singular values in descending order. Take the last $\alpha$ column eigenvectors of left singular vector $\mathbf{U}$, and the dimension is $2 k \times k$. The former $k$ line is the estimation of $\mathbf{d}$, and the latter $k$ line is the estimation of $\mathbf{e}$. Put $\mathbf{d}$ and $\mathbf{e}$ in (17); the obtained $\mathbf{f}_{1}^{*}$ and $\mathbf{f}_{2}^{*}$ are the consistent estimations of the same fault under different conditions.

\section{Fault Prediction Algorithms}

The amplitude changes with the evolution of the fault. When the fault amplitude is estimated, the support vector machine (SVM) prediction model can be used to predict the trend of the fault amplitude.
For the given time series $\left\{x_{1}, x_{2}, \ldots, x_{n}\right\}, i=1,2, \ldots, n$, $x_{n}$ is the target value of prediction, inputs are $\left\{x_{n-1}, x_{n-2}\right.$, $\left.\ldots, x_{n-m}\right\}$, and $m$ is the embedding dimension. Build the mapping $f: \mathbb{R}^{m} \rightarrow F$, which is between input $x_{n}^{*}=\left\{x_{n-1}\right.$, $\left.x_{n-2}, \ldots, x_{n-m}\right\}$ and output $y_{n}=\left\{x_{n}\right\}$, and the learning sample for supporting vector machine is as follows:

$$
X=\left[\begin{array}{cccc}
x_{1} & x_{2} & \cdots & x_{m} \\
x_{2} & x_{3} & \cdots & x_{m+1} \\
\vdots & \vdots & \cdots & \vdots \\
x_{n-m} & x_{n-m+1} & \cdots & x_{n-1}
\end{array}\right], \quad Y=\left[\begin{array}{c}
x_{m+1} \\
x_{m+2} \\
\vdots \\
x_{n}
\end{array}\right]
$$
is

The regression function for training the vector machine

$$
y_{t}=\sum_{i=1}^{n-m}\left(\alpha_{i}-\alpha_{i}^{*}\right) K\left(x_{i}, x_{t}\right)+b, \quad t=m+1, \ldots, n,
$$

where $\alpha_{i}$ and $\alpha_{i}^{*}$ are Lagrange multipliers, $b$ is threshold value, $K\left(x_{i}, x_{t}\right)$ is a Kernel function, and the radial basis function is used here:

$$
K\left(x, x^{\prime}\right)=\exp \left(-\frac{\left\|x-x^{\prime}\right\|^{2}}{2 \sigma^{2}}\right) .
$$

And the one-step prediction model is

$$
y_{n+1}=\sum_{i=1}^{n-m}\left(\alpha_{i}-\alpha_{i}^{*}\right) K\left(x_{i}^{*}, x_{n-m+1}^{*}\right)+b .
$$

And then obtain a sample $x_{n-m+1}^{*}=$ $\left\{x_{n-m+1}, x_{n-m+2}, \ldots, x_{n}\right\}$ and $\widehat{x}_{n+1}$ represents the prediction of the $(n+1)$ th data.

Furthermore we can have

$$
y_{n+p}=\sum_{i=1}^{n-m}\left(\alpha_{i}-\alpha_{i}^{*}\right) K\left(x_{i}^{*}, x_{n-m+p}^{*}\right)+b .
$$

Equation (23) represents the prediction model of the $p$ th step and we can have $x_{n-m+p}^{*}=\left\{x_{n-m+p}, \ldots, \widehat{x}_{n+p}, \widehat{x}_{n+p+1}\right\}, x_{n}$ represents the actual value of the $n$th data, and $\widehat{x}_{n}$ represents the prediction of the $n$th data [31].

Use mean squared error (MSE) and average relative prediction error (ARE) to evaluate the accuracy of prediction of the trend of the fault amplitude using the SVM prediction model. The MSE and ARE can be described as follows:

$$
\begin{gathered}
\text { MSE }=\frac{1}{n} \times \sqrt{\sum_{i=1}^{n}\left(y_{i}-\widehat{y}_{i}\right)^{2},} \\
\text { ARE }=\frac{1}{n} \times \sum\left|\frac{y_{i}-\widehat{y}_{i}}{y_{i}}\right| .
\end{gathered}
$$

\section{Simulations}

Tennessee Eastman process contents 41 measured variables (including 22 continuous variables and 19 component variables) and 12 operating variables and can set 21 kinds of 


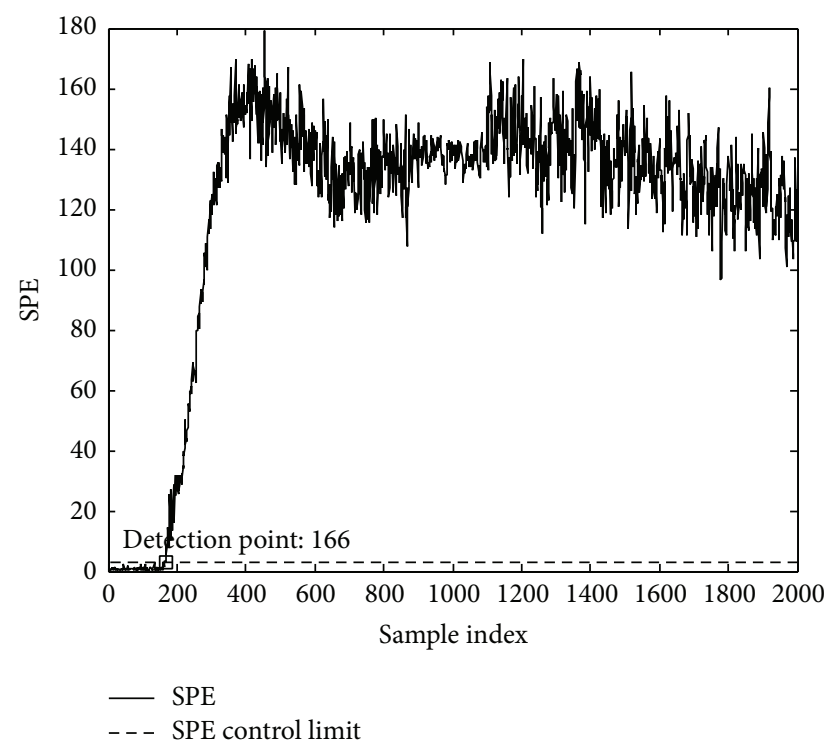

FIGURE 1: The curve of SPE, when the fault occurs at condition 1.

faults; refer to paper [32-35] for more details of Tennessee Eastman process. Simulation of this paper selects fault 2, namely, step transition of flow B. Add a step signal to flow $B$ in a specific instant, and then the variable begins to have a slowly changing process and finally stays at a stable state, so the data of two stable conditions and a transition process can be obtained. Select 11 control variables and 18 process variables as a sample data set, and the sampling interval is 3 minutes, and take 2000 normal samples which contain both working condition 1 , transition process and condition 2 as training samples. Under normal working conditions, changes in working conditions occur at sample point 900 , and, after a period of transition process, the steady state of condition 2 arrives at about sample point 1100 . Under fault conditions, changes in working conditions still occur at sample point 900 , which the time of fault include two cases in Case 1, the fault occurs in condition 1 , as the condition changes and the fault enters the transition process and then lasts to condition 2 , and in this case the fault is set at sample point 160. In Case 2, the fault occurs in condition 2, condition 1 and transition process are in normal state, and, in this case, the fault is set at sample point 1260 .

First, with the multicondition data after pretreatment, the multi-PCA model can be established and set the control limit of statistical indicators for fault detection as $99 \%$. The fault that occurs at both cases has been detected; the detection result of the first case is showed in Figures 1 and 2; the detection result of the second case is showed in Figures 3 and 4 .

In Figure 1, the dash line represents the control limit of SPE and the detection indicator of SPE surpasses the control limit from the 166th sample point that means fault occurs at condition 1.

In Figure 2, the dash line represents the control limit of $T^{2} ; T^{2}$ surpasses the control limit from the 164th sample point that means fault occurs at condition 1.

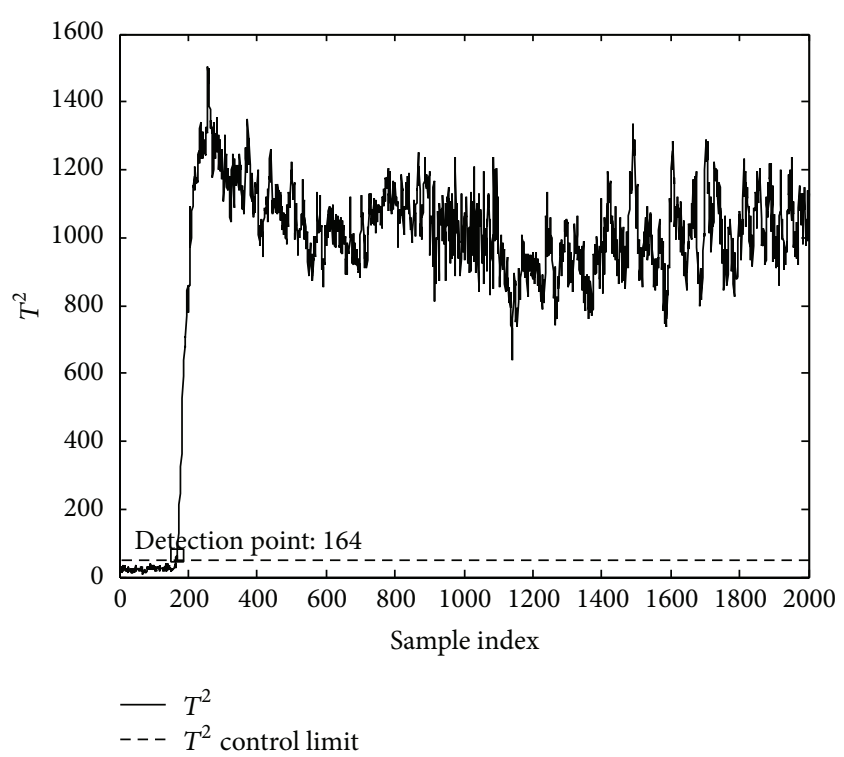

FIgUre 2: The curve of $T^{2}$, when the fault occurs at condition 1.

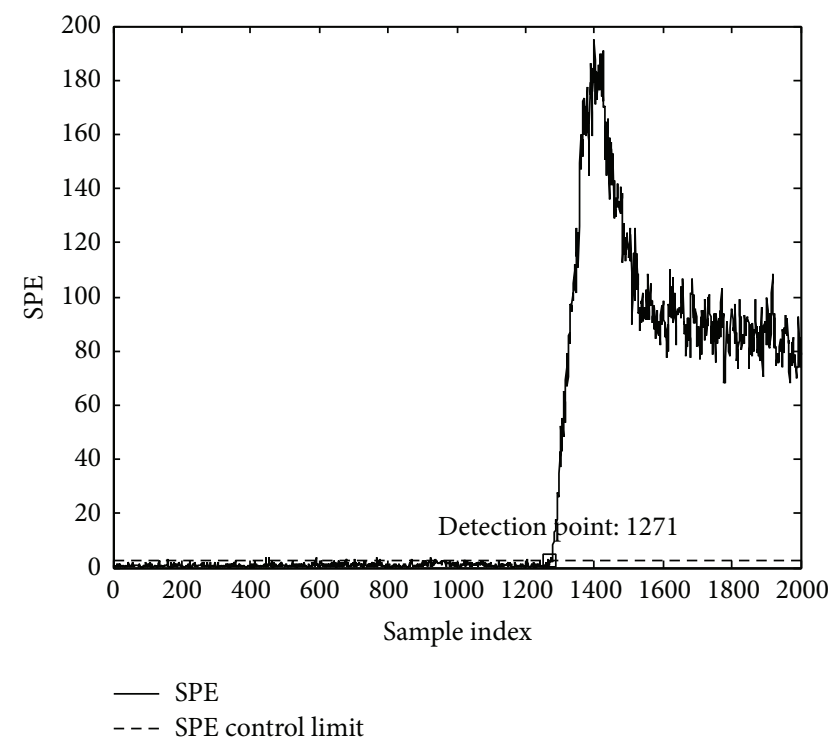

FIGURE 3: The curve of SPE, when the fault occurs at condition 2.

In Figure 3, the dash line represents the control limit of SPE; the detection indicator of SPE surpasses the control limit from the 1271st sample point that means fault occurs at condition 2.

In Figure 4, the dash line represents the control limit of $T^{2} ; T^{2}$ surpasses the control limit from the 1269th sample point that means fault occurs at condition 2 .

After fault detection was accomplished, we can derive the estimation of amplitude of fault using reconstruction technique. Suppose the same fault occurs at two different conditions; then the cure of the estimated amplitude of the fault is shown as in Figure 5. The dot-dash line represents the estimation of amplitude under condition 1, the solid line 


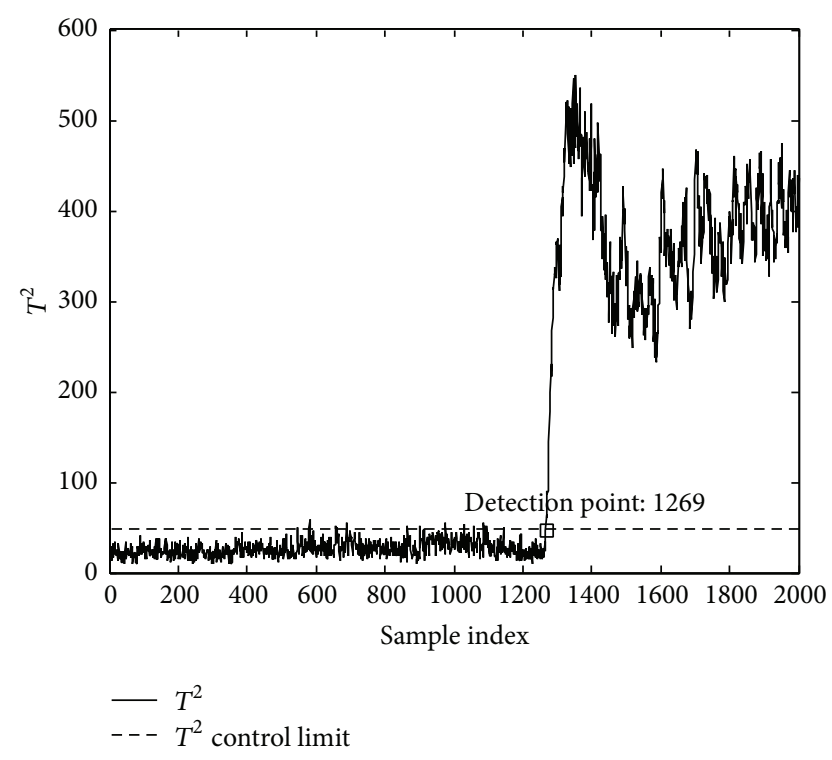

FIGURE 4: The curve of $T^{2}$, when the fault occurs at condition 2.

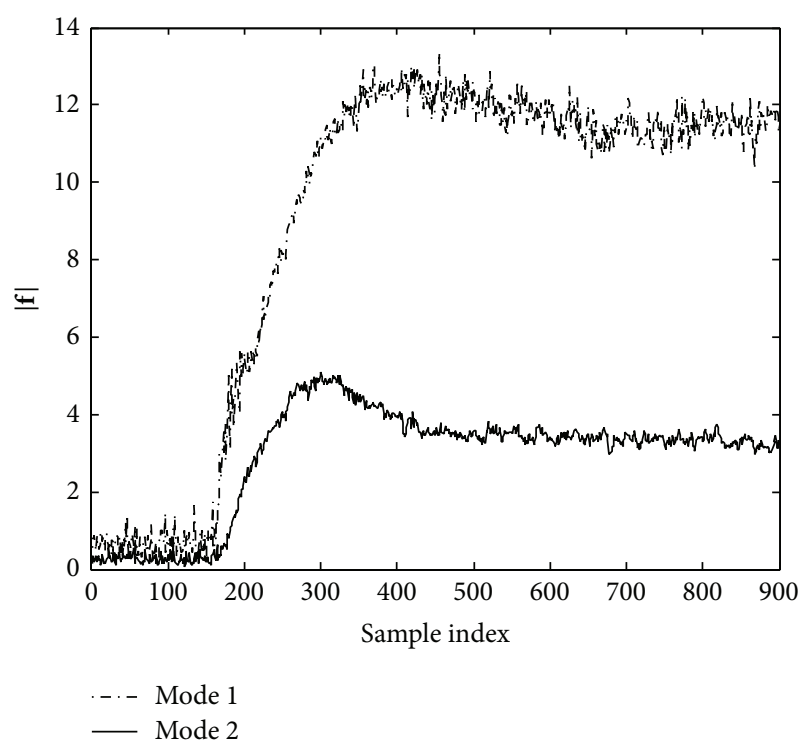

FIGURE 5: Estimation of amplitude of the same fault under two different conditions.

represents the estimation of amplitude under condition 2, and we can easily see that they are not consistent in this situation.

In Figure 6, the consistent estimation of the amplitude of the same fault under two different conditions is derived.

We set that the fault occurs at condition 1 and last to condition 2, the amplitude of the fault is shown in Figure 7, and the consistent estimation of the amplitude is derived by consistent estimation algorithm; see Figure 8.

In Figure 9, employ SVM prediction model to predict the one-step and ten-step amplitude of the fault, the solid line represents the actual value of the amplitude, the dot-dash line represents the one-step prediction curve, and the star line represents the ten-step prediction curve.

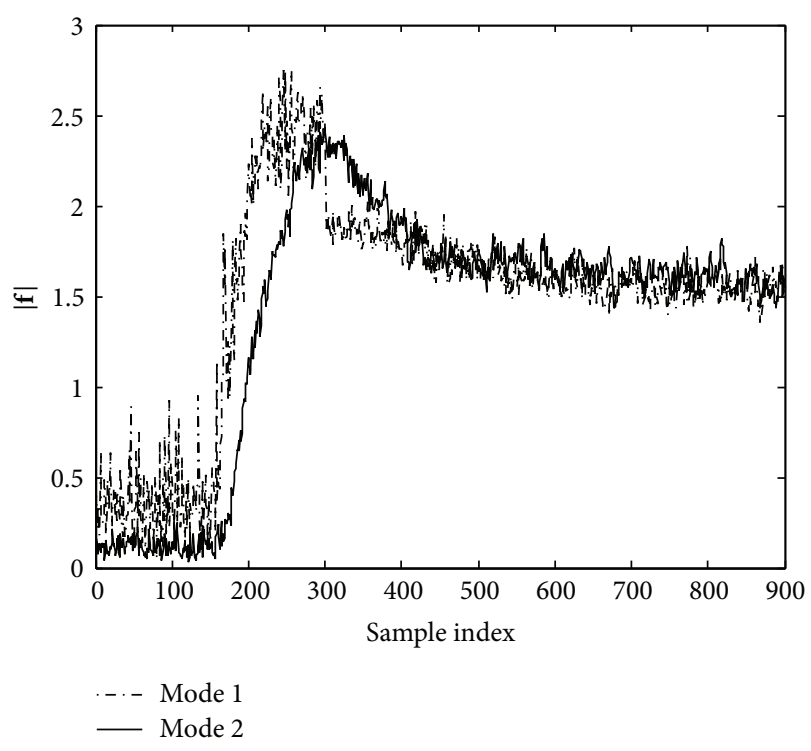

Figure 6: Consistent estimation of the amplitude of the same fault under two different conditions.

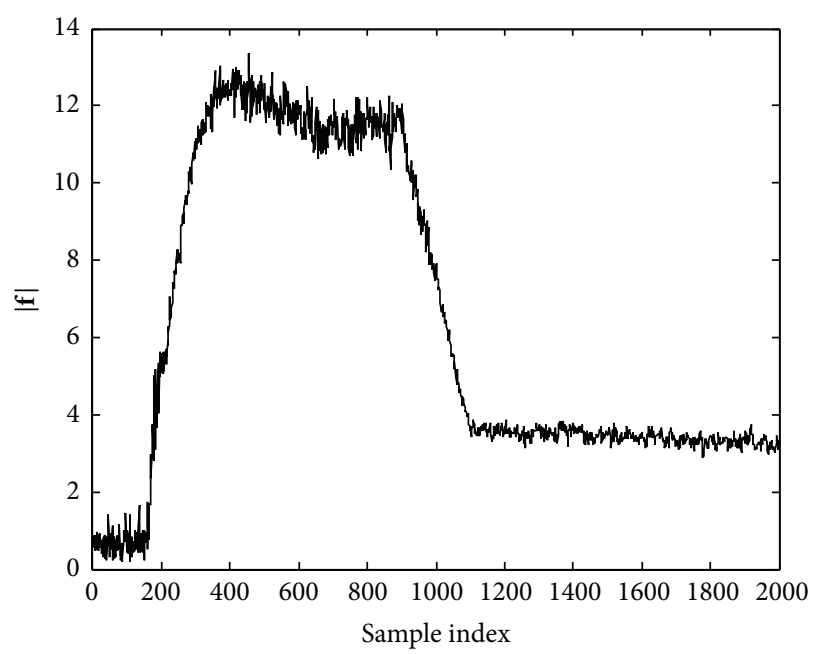

FIGURE 7: Fault occurs at condition 1 and continues to condition 2.

TABLE 1: Comparison of relative prediction errors of different prediction steps.

\begin{tabular}{lc}
\hline Prediction step & Relative prediction error \\
\hline One-step prediction & $2.027 e-008$ \\
Ten-step prediction & 0.00264795 \\
\hline
\end{tabular}

According to the characteristics of multicondition data, SVM prediction model has a good generalization performance; Table 1 shows comparison of relative prediction error of the one-step prediction and 10-step prediction; it can be easily seen that, in the long-term forecast, SVM prediction model still remains high accuracy. 


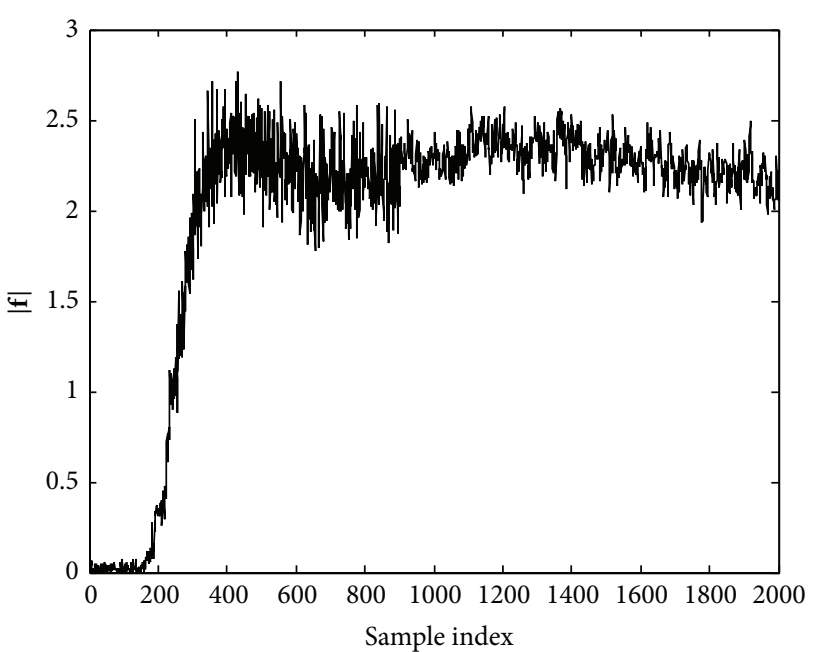

FIGURE 8: The consistent estimation of the amplitude.

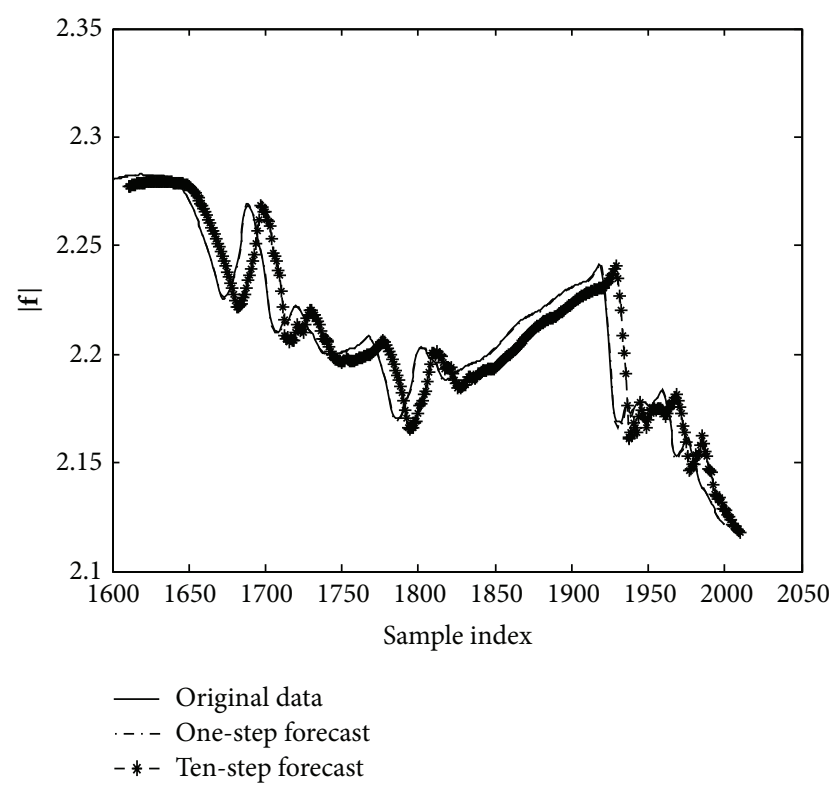

Figure 9: Prediction curve of the amplitude.

\section{Conclusions}

When complex engineering systems run in multiple mode process, the relationship between different measurement variables varies according to the operating mode. If we describe the device status according to a one-condition data model, a lot of false alarms and missing alarms will happen. For a class of multiple mode process with hidden degradation faults, the paper proposes a fault prediction algorithm based on the combination of multi-PCA model and fault reconstruction techniques, which can offer a good solution for fault prediction problems of multiple mode process data. To find the fault direction and estimate the fault amplitude from data with hidden faults, both multidimensional characteristics and consistent estimation of the fault have been considered, and thus the estimation error of the same fault under different conditions can be avoided dramatically. Relatively ideal predictions are obtained by employing SVM to predict the trend of the fault amplitude. By running to Tennessee Eastman process, the effectiveness of reconstruction and prediction algorithms proposed in this paper has been verified.

\section{Conflict of Interests}

The authors declare that there is no conflict of interests regarding the publication of this paper.

\section{Acknowledgments}

This work is supported by National Natural Science Foundation of China (61273173) and National Natural Science Foundation of Beijing (4132009).

\section{References}

[1] Z.-Q. Ge and Z.-H. Song, "New online monitoring method for multiple operating modes process," Journal of Chemical Industry and Engineering, vol. 59, no. 1, pp. 135-141, 2008.

[2] F.-L. Wang, S. Tan, J. Peng, and Y.-Q. Chang, "Process monitoring based on mode identification for multi-mode process with transitions," Chemometrics and Intelligent Laboratory Systems, vol. 110, no. 1, pp. 144-155, 2012.

[3] M. Kano, S. Tanaka, S. Hasebe, I. Hashimoto, and H. Ohno, "Combination of independent component analysis and principal component analysis for multivariate statistical process control," in Proceedings of the International Symposium on Design, Operation and Control of Chemical Plants, pp. 319-324, PSE Asia, 2002.

[4] M. Kano, S. Hasebe, I. Hashimoto, and H. Ohno, "Evolution of multivariate statistical process control: application of independent component analysis and external analysis," Computers and Chemical Engineering, vol. 14, no. 5, pp. 467-485, 2004.

[5] Z. Niu, J.-Z. Liu, and Y.-G. Niu, "Fault detection under varying load conditions based on dynamic multi-principal component models," Journal of Chinese Society of Power Engineering, vol. 25, no. 4, pp. 554-598, 2005.

[6] X.-Y. Wang, X. Wang, Z.-L. Wang, and Q. Feng, "Multiple models external analysis and greedy-KP1M based process monitoring with multiple operation modes," Chemical Industry and Engineering Society of China, vol. 63, no. 9, pp. 2869-2876, 2012.

[7] S. J. Zhao, J. Zhang, and Y. M. Xu, "Monitoring of processes with multiple operating modes through multiple principle component analysis models," Industrial and Engineering Chemistry Research, vol. 43, no. 22, pp. 7025-7035, 2004.

[8] D.-H. Hwang and C. Han, "Real-time monitoring for a process with multiple operating modes," Control Engineering Practice, vol. 7, no. 7, pp. 891-902, 1999.

[9] D. Kim and I.-B. Lee, "Process monitoring based on probabilistic PCA," Chemometrics and Intelligent Laboratory Systems, vol. 67, no. 2, pp. 109-123, 2003.

[10] Y. S. Ng and R. Srinivasan, "An adjoined multi-model approach for monitoring batch and transient operations," Computers \& Chemical Engineering, vol. 33, no. 4, pp. 887-902, 2009.

[11] J.-L. Liu and D.-S. Chen, "Fault detection and identification using modified bayesian classification on PCA subspace," Industrial and Engineering Chemistry Research, vol. 48, no. 6, pp. 3059-3077, 2009. 
[12] J.-L. Liu and D.-S. Chen, "Operational performance assessment and fault isolation for multimode processes," Industrial and Engineering Chemistry Research, vol. 49, no. 8, pp. 3700-3714, 2010.

[13] J.-L. Liu and D.-S. Chen, "Nonstationary fault detection and diagnosis for multimode processes," AIChE Journal, vol. 56, no. 1, pp. 207-219, 2010.

[14] S. J. Qin and J. Yu, "Multimode process monitoring with bayesian inference-based finite Gaussian mixture models," AIChE Journal, vol. 54, no. 7, pp. 1811-1829, 2008.

[15] X.-Z. Xu, L. Xie, and S.-Q. Wang, "Multi-mode process monitoring method based on PCA mixture model," CIESC Journal, vol. 62, no. 3, pp. 743-752, 2011.

[16] N. Lu, F. Gao, and F. Wang, "Sub-PCA modeling and on-line monitoring strategy for batch processes," AIChE Journal, vol. 50, no. 1, pp. 255-259, 2004.

[17] C. Zhao, F. Wang, N. Lu, and M. Jia, "Stage-based soft-transition multiple PCA modeling and on-line monitoring strategy for batch processes," Journal of Process Control, vol. 17, no. 9, pp. 728-741, 2007.

[18] Y. Yao and F.-R. Gao, "Phase and transition based batch process modeling and online monitoring," Journal of Process Control, vol. 19, no. 5, pp. 816-826, 2009.

[19] D.-H. Zhou and Y.-Y. Hu, "Fault diagnosis techniques for dynamic systems," Acta Automatica Sinica, vol. 35, no. 6, pp. 748-758, 2009.

[20] Y. Suresh, L. Kumar, and S. K. Rath, "Statistical and machine learning methods for software fault prediction using CK metric suite: a comparative analysis," ISRN Software Engineering, vol. 2014, Article ID 251083, 15 pages, 2014.

[21] K. Pearson, "On lines and planes of closest fit to systems of points in space," Edinburgh and Dublin Philosophical Magazine and Journal of Science, vol. 6, no. 2, pp. 572-575, 1901.

[22] H. Hotelling, "Analysis of a complex of statistical variables into principal components," Journal of Educational Psychology, vol. 40, no. 8, pp. 1341-1375, 1933.

[23] S. J. Qin, "Statistical process monitoring: basics and beyond," Journal of Chemometrics, vol. 17, no. 8-9, pp. 480-502, 2003.

[24] B. M. Wise and N. B. Gallagher, "The process chemometrics approach to process monitoring and fault detection," Journal of Process Control, vol. 6, no. 6, pp. 329-348, 1996.

[25] R. Dunia, S. J. Qin, T. F. Edgar, and T. J. McAvoy, "Identification of faulty sensors using principal component analysis," AIChE Journal, vol. 42, no. 10, pp. 2797-2811, 1996.

[26] R. Dunia and S. J. Qin, "Subspace approach to multidimensional fault identification and reconstruction," AIChE Journal, vol. 44, no. 8, pp. 1813-1831, 1998.

[27] H. H. Yue and S. J. Qin, "Reconstruction-based fault identification using a combined index," Industrial and Engineering Chemistry Research, vol. 40, no. 20, pp. 4403-4414, 2001.

[28] B. Sun, R. Kang, and J.-S. Xie, "Research and application of the prognostic and health management system," Systems Engineering and Electronics, vol. 29, no. 10, pp. 1762-1767, 2007.

[29] C. F. Alcala and S. J. Qin, "Reconstruction-based contribution for process monitoring with kernel principal component analysis," Industrial and Engineering Chemistry Research, vol. 49, no. 17, pp. 7849-7857, 2010.

[30] J. Ma, Q.-Y. Wang, G. Li, and D.-H. Zhou, "Reconstructionbased fault prognosis for flue gas turbines with independent component analysis," Asia-Pacific Journal of Chemical Engineering, vol. 9, no. 2, pp. 205-213, 2014.
[31] V. N. Vapnik, The Nature of Statiscal Learning Theory, Springer, New York, NY, USA, 1995.

[32] J. J. Downs and E. F. Vogel, "A plant-wide industrial process control problem," Computers and Chemical Engineering, vol. 17, no. 3, pp. 245-255, 1993.

[33] N. L. Ricker, "Decentralized control of the Tennessee Eastman challenge process," Journal of Process Control, vol. 6, no. 4, pp. 205-221, 1996.

[34] L. H. Chiang, E. Russell, and R. D. Braaz, Fault Detection and Diagnosis in Industrial Systems, Springer, London, UK, 2001.

[35] G. Lee, C. H. Han, and E. S. Yoon, "Multiple-fault diagnosis of the Tennessee Eastman process based on system decomposition and dynamic PLS," Industrial and Engineering Chemistry Research, vol. 43, no. 25, pp. 8037-8048, 2004. 


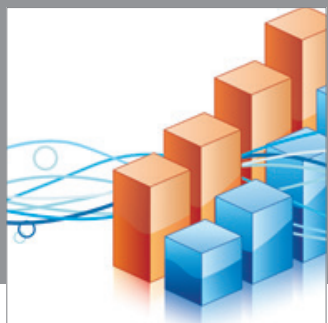

Advances in

Operations Research

mansans

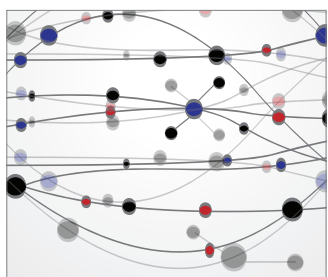

The Scientific World Journal
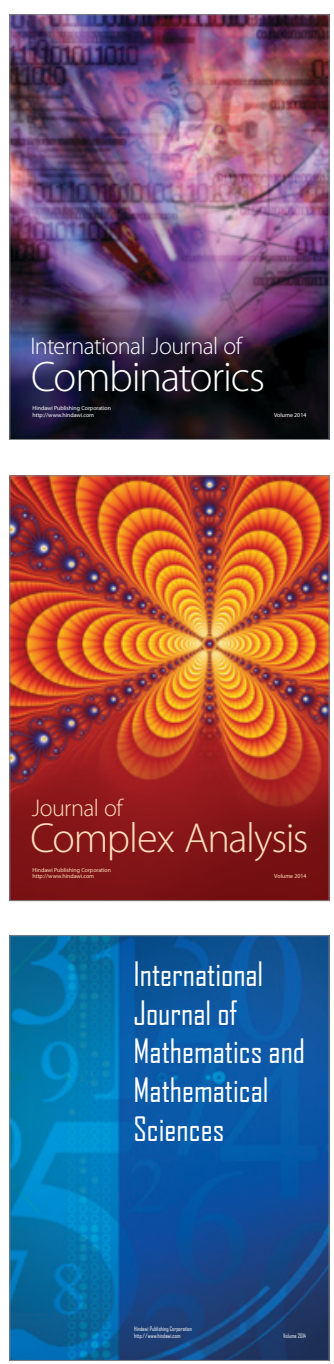
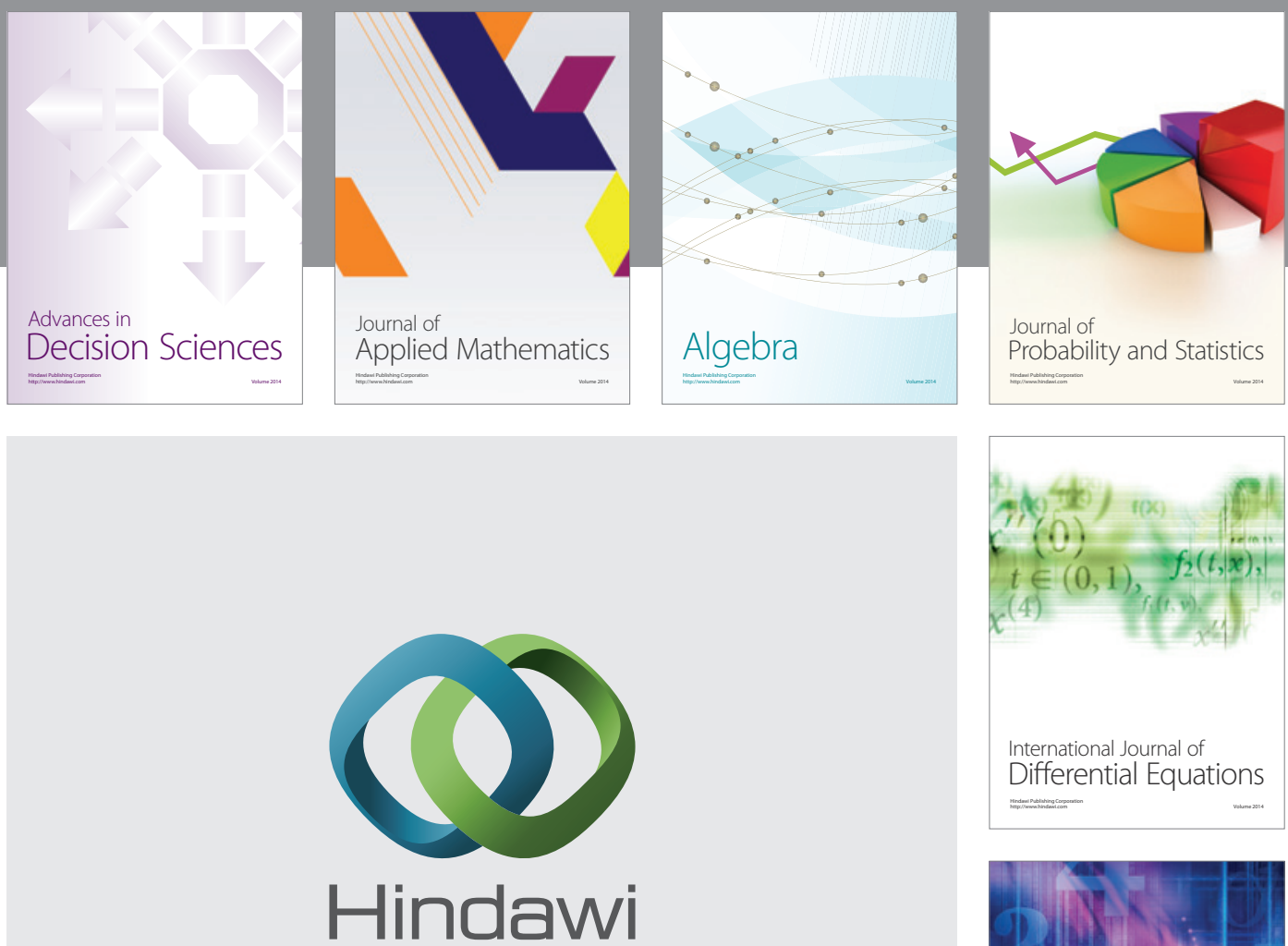

Submit your manuscripts at http://www.hindawi.com
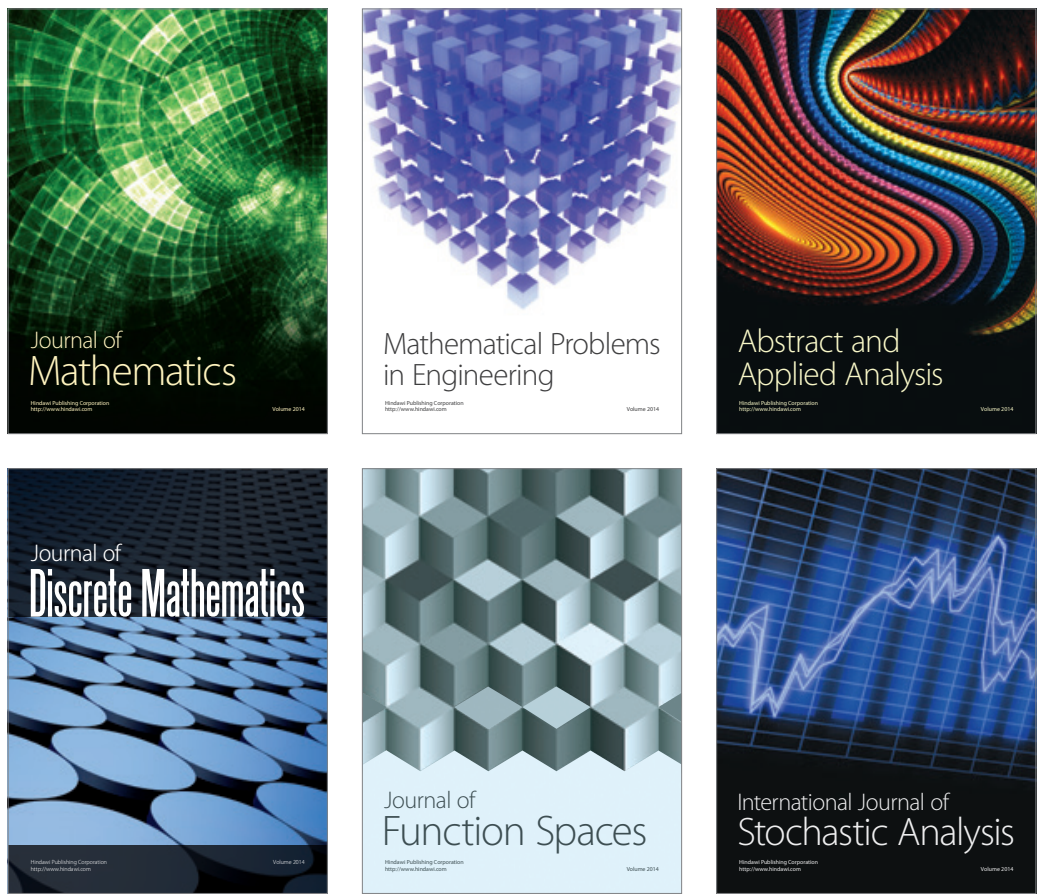

Journal of

Function Spaces

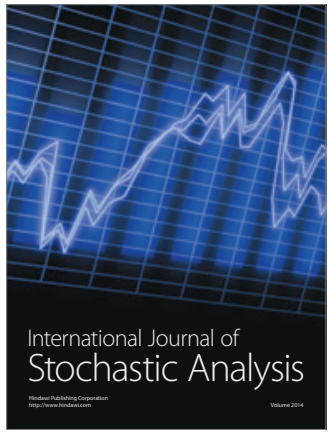

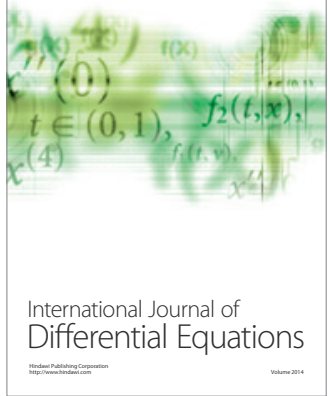
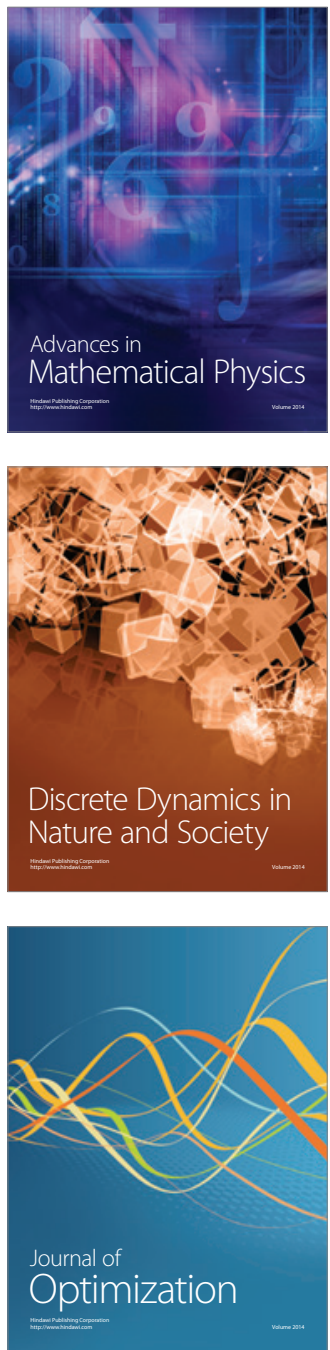